\title{
THE RELATIONSHIP BETWEEN BLOOD PRESSURE AND PUPIL TO LIMBUS DIAMETER RATIO IN HYPERTENSIVE WOMEN: A PILOT STUDY
}

\author{
ARCHANA R ${ }^{1}$, KUMAR SAI SAILESH ${ }^{2 *}$, SRILATHA BASHETTI ${ }^{3}$, SOUMYA MISHRA ${ }^{4}$ \\ ${ }^{1}$ Department of Physiology, Saveetha Medical College, Saveetha University, Chennai, Tamil Nadu, India. ${ }^{2}$ Department of Physiology, DM \\ Wayanad Institute of Medical Sciences, Naseera Nagar, Meppadi (P.0), Wayanad, Kerala, India. ${ }^{3}$ Department of Biochemistry, Apollo \\ Institute of Medical Sciences and Research, Hyderabad, Telangana, India. ${ }^{4}$ Department of Physiology, JIPMER, Pondicherry, India. \\ Email: saisailesh.kumar@gmail.com
}

Received: 11 June 2017, Revised and Accepted: 20 July 2017

\section{ABSTRACT}

Objective: This study was undertaken to observe the relationship between blood pressure (BP) and pupil to limbus diameter (PLD) ratio in hypertensive working women.

Methods: A total of 30 hypertensive working women were part of the study after obtaining written, voluntary, informed consent, and ensuring confidentiality. PLD ratio was measured by two-box method as described in the literature.

Results: Positive correlation was observed between PLD ratio of the right eye ( $\mathrm{r}=0.951)$ and left eye ( $\mathrm{r}=0.927)$ and systolic BP. This correlation is statistically significant at 0.01 levels (two tailed). Positive correlation was observed between PLD ratio of the right eye ( $\mathrm{r}=0.844$ ) and left eye ( $\mathrm{r}=0.828$ ) and diastolic BP. This correlation is statistically significant at 0.01 levels (two tailed). Positive correlation was observed between PLD ratio of the right eye $(r=0.416)$ and left eye $(r=0.396)$ and pulse pressure. This correlation is statistically significant at 0.05 levels (two tailed). Positive correlation was observed between PLD ratio of the right eye $(\mathrm{r}=0.883)$ and left eye $(\mathrm{r}=0.844)$ and mean arterial BP. This correlation is statistically significant at 0.05 levels (two tailed). Positive correlation was observed between PLD ratio of the right eye ( $\mathrm{r}=0.841$ ) and left eye ( $\mathrm{r}=0.819$ ) and pulse rate. This correlation is statistically significant at 0.05 levels (two tailed).

Conclusion: Positive correlation was observed between PLD ratio and autonomic parameters. We recommend further detailed studies to utilize the measurement of PLD ratio as an autonomic function test.

Keywords: Pupil, Limbus, Autonomic parameters, Blood pressure, Hypertension.

(c) 2017 The Authors. Published by Innovare Academic Sciences Pvt Ltd. This is an open access article under the CC BY license (http://creativecommons. org/licenses/by/4. 0/) DOI: http://dx.doi.org/10.22159/ajpcr.2017.v10i11.20528

\section{INTRODUCTION}

According to the $7^{\text {th }}$ report of the Joint National Committee on Prevention, detection, evaluation and treatment of high blood pressure (BP), systolic BP (SBP) 130-139 $\mathrm{mmHg} /$ diastolic BP (DBP) 80-89 $\mathrm{mmHg}$ was included in the category of prehypertension and SBP 140-159 and DBP 90-99 mmHg as stage 1 hypertension [1-3]. In the Women's Health Initiative, black race, poor socioeconomic status, history of cardiovascular disease, physical inactivity, overweight/ obesity, and excess alcohol consumption are considered as factors for the prevalence of hypertension [3]. Furthermore, there is a misconception that women have low chance of heart diseases than men, but hypertension is a major risk factor for cardiovascular diseases in women [4]. As more than half of the hypertensive patients are unaware of the condition, regular monitoring of BP may help in the diagnosis of hypertension [5]. By measuring pupil diameter as an indicator of autonomic nervous function, pupil to limbus diameter (PLD) ratio may correlate with alterations in BP [6]. PLD ratio is defined here as "the ratio of the pupillary diameter measured at an axial plane with the limbal diameter measured at a same or parallel axial plane" [7]. Recent studies show that there is a positive correlation between BP and PLD ratio of right and left eye and significant positive correlation between pulse rate and PLD ratio of the right and left eye [8]. This study was undertaken to observe the relationship between BP and PLD ratio in hypertensive working women.

\section{METHODS}

This study was conducted at the Department of Physiology, Little Flower Institute of Medical Sciences and Research and Department of
Ophthalmology, Little Flower Hospital and Research Centre, Angamaly, from October 2016 to March 2017.

\section{Participants}

A total of 30 hypertensive working women were part of the study after obtaining written, voluntary, informed consent, and ensuring confidentiality. All the participants underwent general physical examination by a qualified female medical officer. The following criteria were followed in recruiting the participants.

\section{Inclusion criteria}

Age between 25 and 50 years, SBP in between 130 and 159 and DBP $80-99 \mathrm{mmHg}, 8 \mathrm{hrs}$ of working, married, willing participants, participants not having any eye diseases, not having any major diseases/ complications, not under any kind of medication/therapy.

\section{Methods}

All measurements were performed at $1 \mathrm{pm}$ for the convenience of the participants and to overcome the effect of diurnal variation.

\section{Measurement of PLD ratio}

PLD ratio was measured by two-box method as described in the literature [7]. PLD ratio was measured at constant illuminance for all the participants. Illuminance measurement for the ambient light conditions was made using a Luxmeter (Model no. MTQ 1010A, Metro Q) with a range of 0-20,000 Lux and resolution of 1-100 Lux. Images were captured using Samsung Galaxy J7. Before capturing the image of the eye, the participants were exposed to the ambient light levels for at least 5 minutes. 
Recording of BP and pulse rate

$\mathrm{BP}$ and pulse rate were recorded using diamonddigital sphygmomanometer (BPDG024) from right hand [9]. Three readings were taken and average value was considered as BP and pulse rate.

\section{Ethical clearance}

The study protocol was approved by Institutional Ethical Committee (EC 27/1/16). Permission obtained from hospital authorities to carry out the study.

\section{Statistical analysis}

Data were analyzed by SPSS 16.0. Statistical test applied are Pearson correlation coefficient and regression analysis. $\mathrm{p}<0.05$ was considered as significant.

\section{RESULTS}

Fig. 1 presents the demographic data of the participants. Positive correlation was observed between PLD ratio of the right eye ( $\mathrm{r}=0.951)$ and left eye ( $\mathrm{r}=0.927)$ and SBP (Fig. 2). This correlation is statistically significant at 0.01 level (two tailed), and unstandardized coefficient (B) obtained by regression analysis is 0.008 for both right and left eye. Positive correlation was observed between PLD ratio of the right eye $(\mathrm{r}=0.844)$ and left eye $(\mathrm{r}=0.828)$ and DBP (Fig. 2). This correlation is statistically significant at 0.01 level (two tailed), and unstandardized coefficient (B) obtained by regression analysis is 0.008 for both right and left eye. Positive correlation was observed between PLD ratio of the right eye $(r=0.416)$ and left eye $(r=0.396)$ and pulse pressure (Fig. 3). This correlation is statistically significant at 0.05 level (two tailed), and unstandardized coefficient (B) obtained by regression

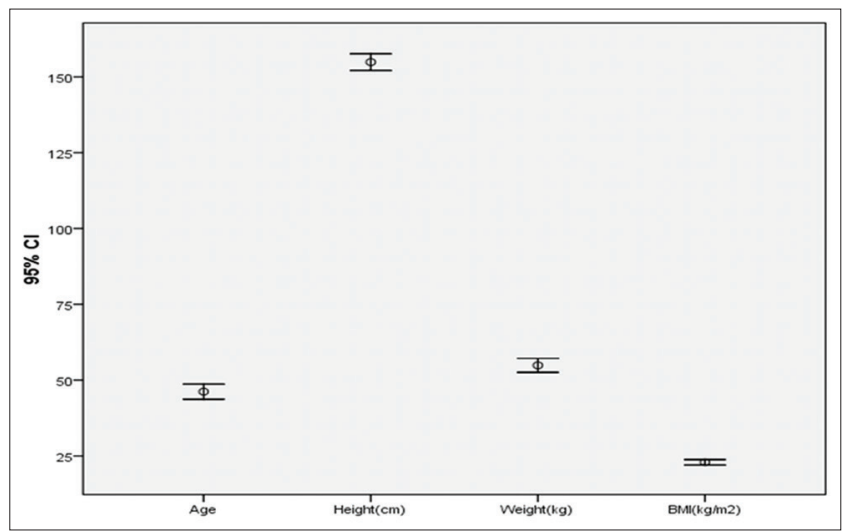

Fig. 1: Demographic data of the participants analysis is 0.006 for both right and left eye. Positive correlation was observed between PLD ratio of the right eye $(\mathrm{r}=0.883)$ and left eye $(\mathrm{r}=0.844)$ and mean arterial BP (Fig. 3). This correlation is statistically significant at 0.05 level (two tailed), and unstandardized coefficient (B) obtained by regression analysis is 0.009 for both right and left eye. Positive correlation was observed between PLD ratio of the right eye $(\mathrm{r}=0.841)$ and left eye $(\mathrm{r}=0.819)$ and pulse rate (Fig. 4). This correlation is statistically significant at 0.05 level (two tailed), and unstandardized coefficient (B) obtained by regression analysis is 0.005 for both right and left eye.

\section{DISCUSSION}

Hypertension is considered as a major health problem worldwide. In 2008 , WHO rated $40 \%$ of people above the age of 25 years as hypertensive [10]. In 2010, hypertension was attributed as an important risk factor for many diseases in the South Asia [11]. Hypertension plays a leading role in the cause of premature death and disability [12]. Furthermore, it is directly linked to the risk of developing cardiovascular diseases, cerebrovascular diseases, and chronic kidney diseases [13-16]. Studies reveal that $33 \%$ urban and $25 \%$ of Indians are hypertensive and only $1-10^{\text {th }}$ of rural and $1-5^{\text {th }}$ of urban are on control [17]. Hypertension was described as a syndrome and was considered as a major public health issue, and it is called as silent killer disease [18,19].

Pupil located in the center of the eye play a key role in regulating the light entry, and it quickly responds to modulations in the surrounding environment $[20,21]$. Variations in the pupil diameter are regulated by two muscles, the sphincter and dilator which are innervated by the parasympathetic nervous system and sympathetic nervous system simultaneously [22]. Pupil dilates through sympathetic stimulation by the release of adrenaline and parasympathetic inhibition [23,24]. Recent studies show that there is positive correlation between BP and PLD ratio and a strong correlation between PLD ratio and pulse rate in healthy women [8]. In our current study, we observe a strong correlation between PLD ratio of right and left eye with BP and pulse rate. The diameter of pupil and BP both are influenced by sympathetic nervous system so the observed correlation may be due to this fact.

\section{CONCLUSION}

We have observed a significant positive correlation between PLD ratio and autonomic parameters. We recommend further detailed studies to recommend the measurement of PLD ratio as an autonomic function test.

\section{ACKNOWLEDGMENT}

Our sincere thanks Rev Fr. Dr. George MD, Principal, Little Flower Institute of Medical Sciences and Research, for his immense support
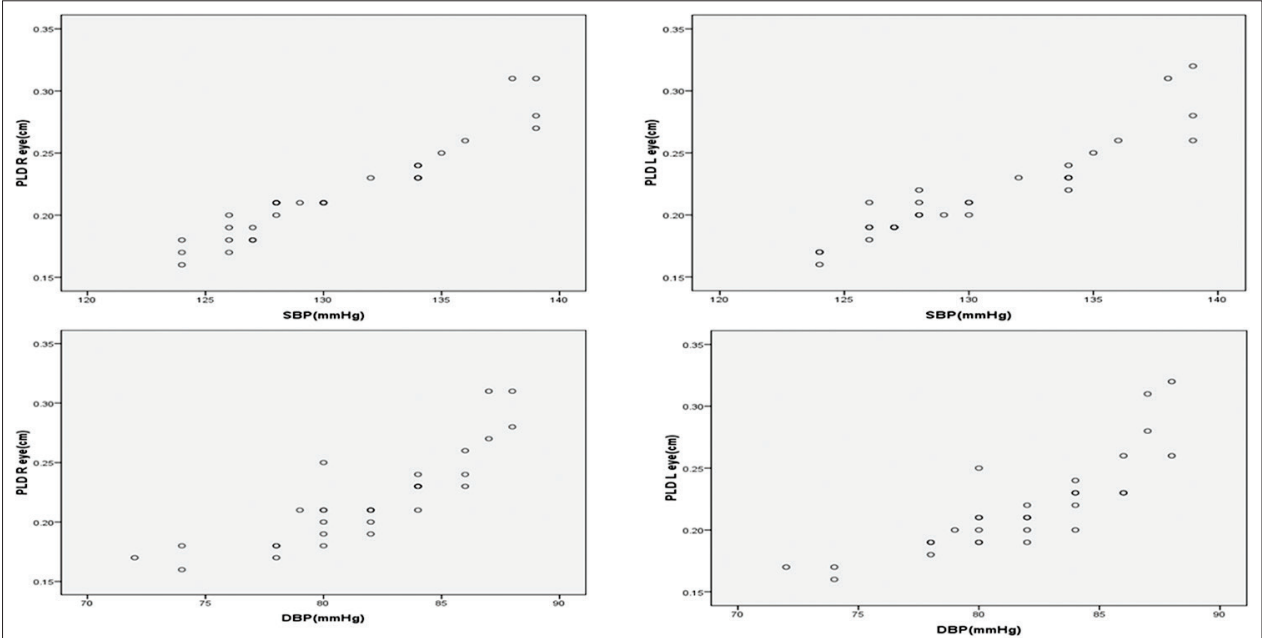

Fig. 2: Association of pupil to limbus diameter ratio of right and left eye with systolic and diastolic blood pressure 

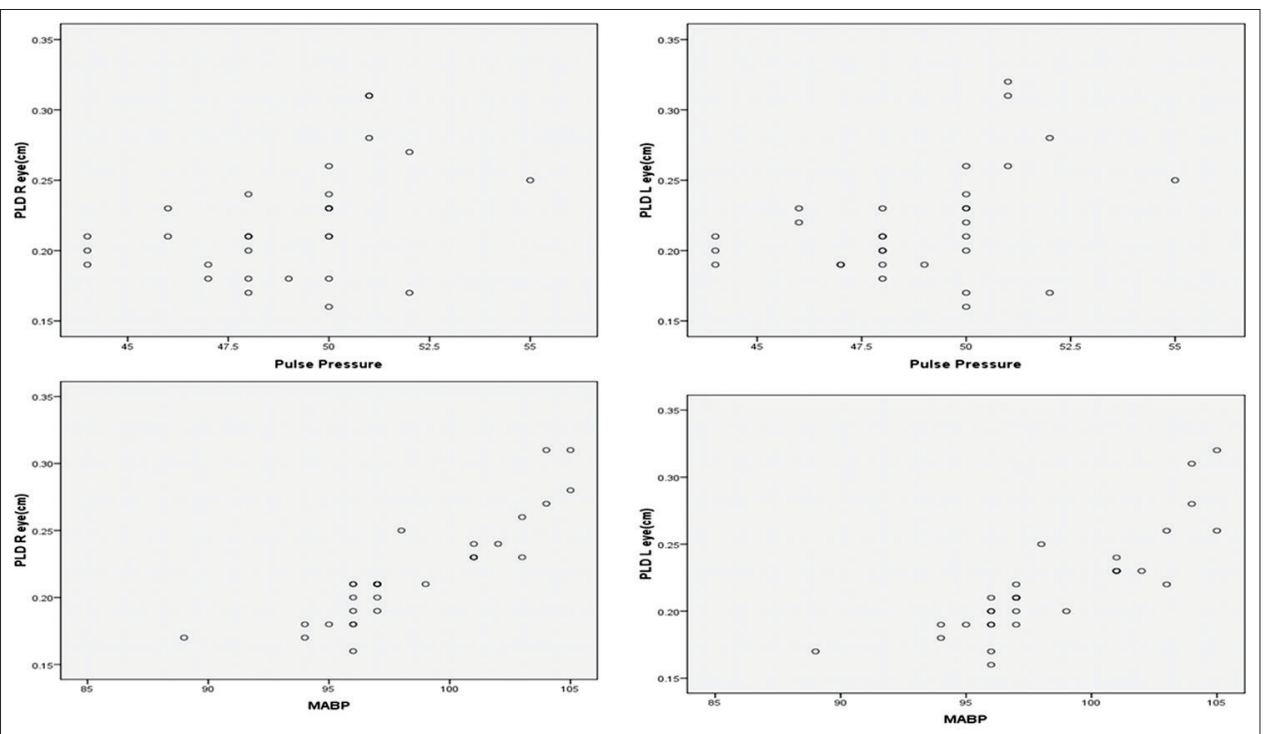

Fig. 3: Association of pupil to limbus diameter ratio of right and left eye with pulse pressure and mean arterial blood pressure
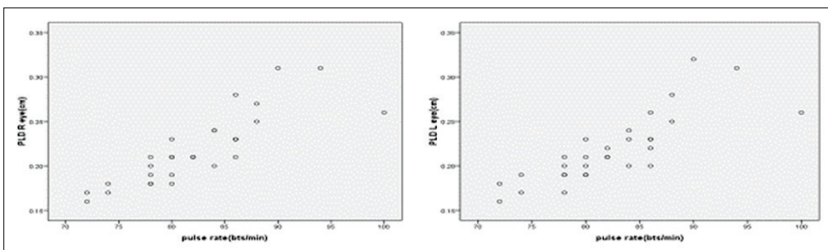

Fig. 4: Association of pupil to limbus diameter ratio of right and left eye with pulse rate

and encouragement throughout the study. We are also thankful to management of Little Flower Hospital and Research Centre for arranging necessary facilities to carry out the study.

\section{REFERENCES}

1. FrancoEmanuele Di AngelantonioDorairaj PrabhakaranJ HypertensChobanian AV, Bakris GL, Black HR, Cushman WC, Green $\mathrm{LA}$, Izzo JL Jr, et al. Seventh report of the joint national committee on prevention, detection, evaluation, and treatment of high blood pressure. Hypertension 2003;42(6):1206-52.

2. Chobanian AV, Bakris GL, Black HR, Cushman WC, Green LA, Izzo JL Jr, et al. The seventh report of the joint national committee on prevention, detection, evaluation, and treatment of high blood pressure: The JNC 7 report. JAMA 2003;289(19):2560-72.

3. Wassertheil-Smoller S, Anderson G, Psaty BM, Black HR, Manson J, Wong $\mathrm{N}$, et al. Hypertension and its treatment in postmenopausal women: Baseline data from the Women's Health Initiative. Hypertension 2000;36(5):780-9

4. Gudmundsdottir H, Høieggen A, Stenehjem A, Waldum B, Os I. Hypertension in women: Latest findings and clinical implications. Ther Adv Chronic Dis 2012;3(3):137-46.

5. Gulec S. Early diagnosis saves lives: Focus on patients with hypertension. Kidney Int Suppl 2013;3(4):332-4.

6. Yamanaka K, Kawakami M. Convenient evaluation of mental stress with pupil diameter. Int J Occup Saf Ergon 2009;15(4):447-50.

7. Mojumder DK, Patel S, Nugent K, Detoledo J, Kim J, Dar N, et al. Pupil to limbus ratio: Introducing a simple objective measure using two box method for measuring early aniscoria and progress of pupillary change in the ICU. J Neurosci Rural Pract 2015;6(2):208-15.

8. Sajeevan A, Sailesh KS. Correlation of pupil to limbus diameter ratio with blood pressure and pulse rate. Int J Pharm Bio Sci 2017;8(3):B12-6.

9. Kumar SS, Rajagopalan A, Mukkadan JK. Vestibular stimulation for stress management in students. J Clin Diagn Res 2016;10(2):CC27-31.

10. World Health Organization. Global Brief on Hypertension. Geneva:
World Health Organization; 2013.

11. Lim SS, Vos T, Flaxman AD, Danaei G, Shibuya K, Adair-Rohani H, et al. A comparative risk assessment of burden of disease and injury attributable to 67 risk factors and risk factor clusters in 21 regions, 1990-2010: A systematic analysis for the Global Burden of Disease Study 2010. Lancet 2012;380(9859):2224-60

12. O'Brien E, Pickering T, Asmar R, Myers M, Parati G, Staessen J, et al. Working group on blood pressure monitoring of the European society of hypertension international protocol for validation of blood pressure measuring devices in adults. Blood Press Monit 2002;7(1):3-17.

13. Qureshi AI, Suri MF, Kirmani JF, Divani AA, Mohammad Y. Is prehypertension a risk factor for cardiovascular diseases? Stroke 2005;36(9):1859-63.

14. Wu S, Huang Z, Yang X, Li S, Zhao H, Ruan C, et al. Cardiovascular events in a prehypertensive Chinese population: Four-year follow-up study. Int J Cardiol 2013;167(5):2196-9.

15. Huang Y, Wang S, Cai X, Mai W, Hu Y, Tang H, et al. Prehypertension and incidence of cardiovascular disease: A meta-analysis. BMC Med 2013;11:177.

16. Kim MJ, Lim NK, Park HY. Relationship between prehypertension and chronic kidney disease in middle-aged people in Korea: The Korean genome and epidemiology study. BMC Public Health 2012;12:960.

17. Anchala R, Kannuri NK, Pant H, Khan H, Franco OH, Di Angelantonio E, et al. Hypertension in India: A systematic review and meta-analysis of prevalence, awareness, and control of hypertension. J Hypertens 2014;32(6):1170-7.

18. Venkataraman R, Kumar BP, Kumaraswamy M, Singh R, Pandey M, Tripathi P, et al. Smoking, alcohol and hypertension. Int J Pharm Pharm Sci 2013;5(4):28-32.

19. Dhianawaty DD, Heryaman H, Syamsunarno MR. Blood pressure profiles among east bongas and west bongas people in effort and support from universitas Padjadjaran and the regent of Majalengka regency and chieves of the villages. Int J Pharm Pharm Sci 2017;9(6):215-9.

20. Oyster CW, editor. The Iris and the pupil. The Human Eye: Structure and Function. Sunderland, Mass: Sinauer Associates; 1999. p. 411-46.

21. Beatty J, Lucero-Wagoner B. The pupillary system. In: Cacioppo JT, Tassinary LG, Berntson GG, editors. Handbook of Psychophysiology. Cambridge, UK: Cambridge University Press; 2000. p. 14-62.

22. Walker HK, Hall WD, Hurst JW. Clinical Methods: The History, Physical, and Laboratory Examinations. $3^{\text {rd }}$ ed. Ch. 58. Boston, MA: Butterworths; 1990.

23. Bradley MM, Miccoli L, Escrig MA, Lang PJ. The pupil as a measure of emotional arousal and autonomic activation. Psychophysiology 2008;45(4):602-7

24. Fotiou F, Fountoulakis KN, Goulas A, Alexopoulos L, Palikaras A. Automated standardized pupillometry with optical method for purposes of clinical practice and research. Clin Physiol 2000;20(5):336-47. 